\title{
Ball Aerospace 4-6 K Space Cryocooler
}

\author{
D. Glaister ${ }^{1}$, W.J. Gully ${ }^{1}$, P. Hendershott ${ }^{1}$, E.D. Marquardt ${ }^{1}$, \\ V. Kotsubo ${ }^{1}$, and R.G. Ross Jr. ${ }^{2}$ \\ ${ }^{1}$ Ball Aerospace \& Technologies Corp. \\ Boulder, CO 80301 \\ ${ }^{2}$ Jet Propulsion Laboratory \\ Pasadena, CA 91109
}

\begin{abstract}
This paper describes the design, development, testing, and performance at Ball Aerospace of a longlife, 4-6 K temperature space cryocooler. For temperatures to $6 \mathrm{~K}$ and below, Ball has developed a hybrid Stirling/J-T (Joule-Thomson) cooler. The hybrid cooler has been verified in test to $3.5 \mathrm{~K}$ on a Ball program to study the J-T system, and a complete system test with the Stirling precooler and J-T cooling loop has been completed on the NASA/JPL Advanced Cryocooler Technology Development Program (ACTDP). This system test has shown that the Ball 4-6 K cooler can meet all the requirements of the James Webb Space Telescope (JWST) Mid-Infrared Instrument (MIRI) cooler including a minimum $70 \mathrm{~mW}$ at $6 \mathrm{~K}$ and $77 \mathrm{~mW}$ at $18 \mathrm{~K} .{ }^{1}$ The ACTDP cooler provides simultaneous cooling at $6 \mathrm{~K}$ (typically for either doped Si detectors or as a precooler for a sub-Kelvin cooler) and $18 \mathrm{~K}$ (typically for optics or shielding) with cooling stages also available at 40 and $180 \mathrm{~K}$ (typically for thermal shields or other components). In the past year, the ACTDP cooler has become the baseline cooling method for the NASA JWST MIRI mission in addition to uses on several other future NASA missions. The $4-6 \mathrm{~K}$ Cooler is highly leveraged off previous Ball space coolers, including multiple life tests and flight units.
\end{abstract}

\section{INTRODUCTION}

Although a number of long-life space coolers have been developed and flown over the past decade, none have been able to provide cooling at $10 \mathrm{~K}$ or below. Most of the deployed systems are regenerative cycle coolers based on the linear "Oxford" approach with noncontacting armatures suspended on diaphragm springs to give them long life. Because they operate at relatively high frequencies ( 30 to $60 \mathrm{~Hz}$ ), it has been difficult to extend their performance to low temperatures because of regenerator limitations.

Ball developed a "hybrid" cryocooler system to get around this specific problem. In this approach the regenerative cooler precools a second J-T recuperative cycle cooler that provides the last stage of cooling. The configuration is depicted in Figure 1. The mature Stirling precooler provides most of the refrigeration, including any auxiliary intermediate temperature cooling required by the system as well as the cooling that prepares the J-T gas for its expansion. The J-T compressor drives a fluid loop consisting of several heat exchangers, heat sinks, and a passive expansion valve. Precooled to $15 \mathrm{~K}$, our J-T coolers have easily reached 5 to $10 \mathrm{~K}$ with ${ }^{4} \mathrm{He}$ and $3.5-5 \mathrm{~K}$ with ${ }^{3} \mathrm{He}$. This modest reduction in final temperature is the key to our long life because it can be achieved with a relatively low pressure ratio that ensures that the J-T compressor will be lightly loaded and will not wear. 


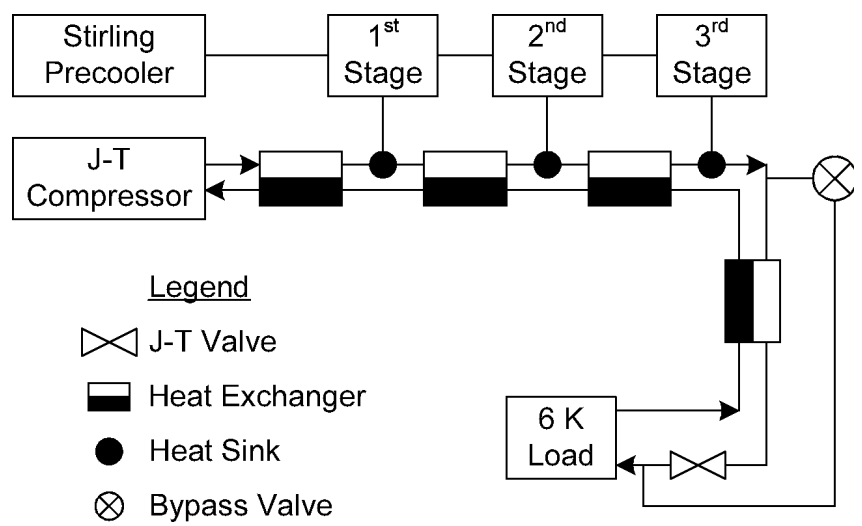

Figure 1. The hybrid cooler consists of a recuperative J-T system precooled by a Stirling cycle mechanical refrigerator.

This paper describes Ball's latest hybrid cooler, its test rig, and the results of our system test. The cooler exceeded the program requirements for the JWST MIRI, specifically $70 \mathrm{~mW}$ at $6.2 \mathrm{~K}$ plus $77 \mathrm{~mW}$ at $18 \mathrm{~K}$ to represent line parasitics on our extended deployment. In 2003, Ball presented performance results for a prototype hybrid consisting of a breadboard J-T system precooled by a commercial GiffordMcMahon (G-M) refrigerator. ${ }^{2}$ The new system is far more representative with new electronics, an advanced J-T, and Ball's latest Stirling cycle refrigerator configured into a complete flight-like system.

\section{CUSTOM TEST SETUP FOR CHARACTERIZING THE MIRI COOLER}

The characterization of the MIRI cooler is subtle because its performance is dominated by parasitic rather than interface heat loads. Only $15 \%$ of the work the cooler does is associated with the load applied at the $6.2 \mathrm{~K}$ interface. The parasitics have to be either reproduced accurately by the test set or eliminated entirely and replaced by a surrogate electrical load at a suitable interface. The test apparatus in Figure 2 was designed and built especially to characterize this cooler.

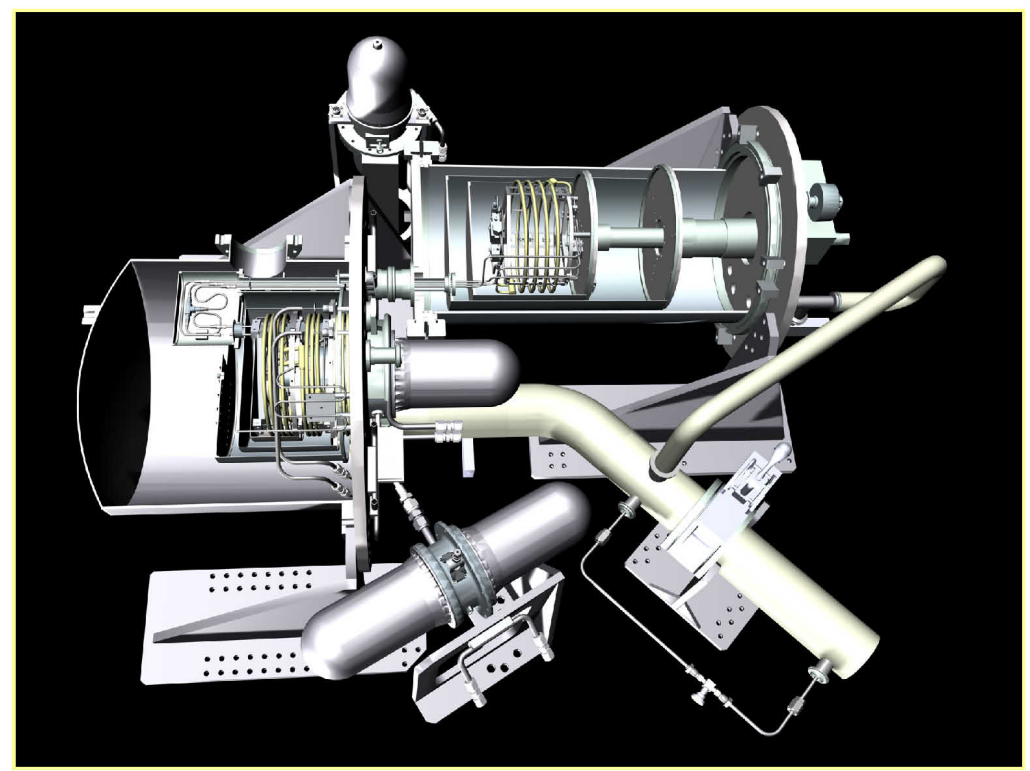

Figure 2. The apparatus for testing the MIRI space cooler has a local chamber representing the bus and a remote chamber representing the instrument environment. 
The apparatus consists of two separate but interconnected chambers reflecting MIRI's split configuration where the active coolers are located on the spacecraft bus and the cold head is located on a remote structure $20 \mathrm{~m}$ away. Our cold head is in the upper chamber in Figure 2, which has a G-M refrigerator and radiation shields for eliminating the parasitics on the components within. Our compressor cold head and its active refrigerators are in the lower chamber. The chambers are connected by a small neck and share a common vacuum. Extensions of the G-M's shields run through the neck to provide a suitable environment for the two refrigerant lines that run between the chambers. The extensions can be seen in Figure 3.

The upper chamber has been designed to shield the remote cold head from the parasitics so its performance can be determined by adding electrical loads to heater interfaces at 6.2 and $18 \mathrm{~K}$ as depicted in Figure 1. Actually, about $10 \%$ of the $18 \mathrm{~K}$ parasitics were due to the tubing supports in the neck region. The parasitics there were monitored and accounted for by measuring the temperatures of both lines on both ends of the span.

The lower chamber contains the very important compressor cold head. Refrigerating the cold head accounts for about two-thirds of the work done by the cooler so it must be truly representative for an accurate characterization to be made. It consists of four parts: a central cold finger support, counterflow heat exchangers, radiation shields, and a tripod support structure. Each is an advanced design and is as light and compact as possible. Since the cold head's thermal performance depends on how these subsystems interact, the cold head was built and thoroughly vibration tested in advance of this test to ensure that subsequent changes would not invalidate our results. Finally, the vacuum can had a cold shroud whose temperature profile could be adjusted to replicate the thermal environment expected on the MIRI bus. External heat rejection temperatures were set and stabilized by fluid chillers.

\section{DESCRIPTION OF THE COOLER COMPONENTS}

An important aspect of this test is that the hardware is relatively mature, with hardware at or near Technology Readiness Level (TRL) of 6 . The two active coolers, the J-T and the Stirling, can be seen in Figure 4.

The J-T linear compressor is identical to that flown on the High Resolution Dynamics Limb Sounder (HIRDLS) instrument, but in this case it is equipped with reed valves for rectifying the pressure. The reed valves are the same as those used by Rutherford Appleton Laboratories (RAL) on the PLANCK instrument, although the flow passages have been resized for the higher flow rates in this application. At this time the HIRDLS compressor has in excess of 16,000 hours on-orbit operation. The J-T compressor creates a pressure head that drives the gas through a piping system. It consists of a passive gas purifier, a set of

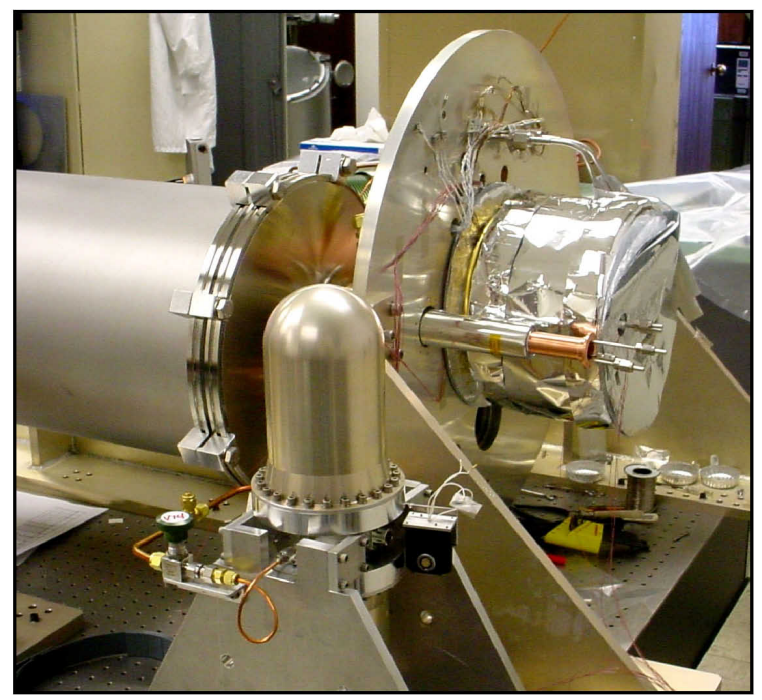

Figure 3. The compressor cold head and the bridge connecting the two chambers. 


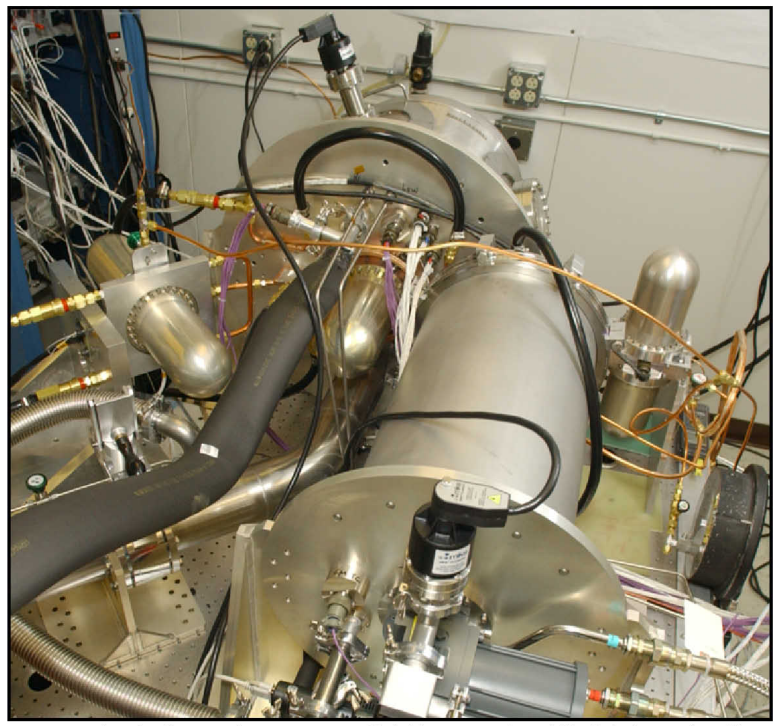

Figure 4. The test set includes a three-stage Stirling cooler (SB315) and a linear compressor equipped with reed valves to drive the J-T loop.

multi-tube-in-tube heat exchangers, precooler heat sinks along the compressor's cold head, and crossover lines to the remote chamber that has the last $(15$ to $6.2 \mathrm{~K})$ heat exchanger, the expansion valve, and a cold tip. ${ }^{3}$ Calibrated "fluid thermometers" for characterizing the performance of the system were mounted in separate, floating heat exchangers at key spots along the J-T lines. Because of its importance, the last exchanger has them at each entrance and exit enabling careful measurements of its effectiveness.

The cold tip consists of an instrumented copper plate with internal surface area for good contact to the helium. Following JPL, the expansion valve was made from a Mott Corporation flow restrictor although sized for this application. Because of the low pressure ratios involved, the flow characteristics of these small sintered impedances can be determined in advance by simple room temperature tests.

The compressor cold head has three enclosures, at 150, 45, and $15 \mathrm{~K}$, supported by a stiff tripod structure. The nested layers enclose the single central displacer cold finger. The warmest stage has a radiation shield and a thick MLI blanket for reflecting the high radiation loads from ambient. The counterflow heat exchangers, suspended from the tripod, are also heat sunk at each stage. The tripod is designed for maximum stiffness with minimum conduction. Each stage is heat sunk to its corresponding Stirling expansion stage through a fluid link. By measuring the flow and the temperature difference between them, the heat transferred, which in equilibrium equals the net load on the stage, can be determined.

The SB315 three-stage $15 \mathrm{~K}$ Stirling cooler is the cornerstone of our system. It consists of a custom three-stage displacer coupled with our high capacity Stirling compressor. The components can be seen to center left in Figure 4. The compressor is relatively new having been developed for our latest two-stage $35 \mathrm{~K}$ cooler, capable of more than $2 \mathrm{~W}$ at $35 \mathrm{~K}$ and $12 \mathrm{~W}$ at $85 \mathrm{~K} .{ }^{4}$ The cooler's mechanical design is backed up by substantial life test hours on similar Stirling coolers like the SB230, SB335, and SB235. The three-stage displacer has expansion stages at 150,45 , and $15 \mathrm{~K}$ to efficiently absorb the parasitic loads. Its last stage is equipped with an advanced version of the rare earth regenerator developed on a NASA Explorer program enabling it to cool to temperatures below $10 \mathrm{~K} .^{5}$

Both coolers were driven by a single set of microprocessor-controlled electronics capable of active vibration control in three axes and of temperature control of the entire system. Although the analog and digital sections are complete, rack-mounted commercial DC supplies were used to provide the de power to the system.

\section{TEST RESULTS}

The main test result is the cooler's refrigeration capacity at $6.2 \mathrm{~K}$ as a function of the J-T compressor stroke. In this test a temperature controller was used to keep the cold tip at $6.2 \mathrm{~K}$, and the $18 \mathrm{~K}$ load was 
held nearly constant at $77 \mathrm{~mW}$. The precooler temperature was held near $16 \mathrm{~K}$. Results for our thermodynamic model for a given precooler temperature, flow, and heat exchanger effectiveness are in good agreement with the cooler's heat lift as shown in Figure 5. The cooler carried a minimum of $75 \mathrm{~mW}$ at $6.2 \mathrm{~K}$ (vs. a requirement of 66) as well as up to $77 \mathrm{~mW}$ of cooling at $18 \mathrm{~K}$ (vs. a requirement of 70). The loads are mostly independent of each other, and the $6.2 \mathrm{~K}$ load can be varied over a wide range simply by changing the J-T compressor's stroke. The projected system input power is also given. As mentioned, part of the power chain was commercial so a corrected total input power, representative of a $28 \mathrm{Vdc}$ flight set of electronics, has been provided. The projections are based on measured component powers and the estimated performance of the flight electronics.

In MIRI, the bulk of the parasitics fall on the plumbing that lies between the compressor cold head and the fourth heat exchanger. As long as they are not too large, they cause only a slight rise in gas temperature and do not interfere with the J-T's performance. The loads are substantially independent and enter the precooler at $16 \mathrm{~K}$. Note the advantage over a hypothetical $6.2 \mathrm{~K}$ cooler using a circulator to transfer cooling to the remote location because both loads would have to be supplied at the lower temperature, which would be fundamentally less efficient.

The system performance hinges on the isolation provided by the fourth counterflow heat exchanger. More than $1 \mathrm{~W}$ of sensible heat has to be transferred from the inward bound stream to the outward bound stream, and as the cooling power is only $75 \mathrm{~mW}$, the exchanger has to be nearly ideal for the system to work well. The heat exchanger temperatures on both ends were carefully monitored so its effectiveness could be independently gauged. The effectiveness is the ratio of the heat transferred to the maximum heat that could be transferred, Qactual/Qmax; these quantities were determined from temperature and pressure measurements. Results for two different exchangers that served in that location are shown in Figure 6. The first is for the 2002 exchanger that was initially used to debug the apparatus; it was not very effective. The intended exchanger, sized for the desired flow rate, approached $99.5 \%$ effectiveness at its design mass flows. This outstanding result was a contributing factor to the solid system performance.

Measurements of the compressor cold head's parasitic loads were also made. Each of its three stages had a mix of radiative, conductive, and fluid loads. At equilibrium the net load was transferred to the precooler through a calibrated thermal link so it could be measured. The loads on each stage at the normal operating point are shown in Figure 7. The individual contributions could be backed out by varying the flow rate and the stage temperatures. The results agree reasonably well with our math models. The only deficiency had to do with how the radiation from the internal snubber tube split between the second and third stages.

The simple J-T system depicted in Figure 1 has difficulty starting from a high temperature because the J-T expansion valve's flow resistance increases with temperature. With low flow the refrigerating power is

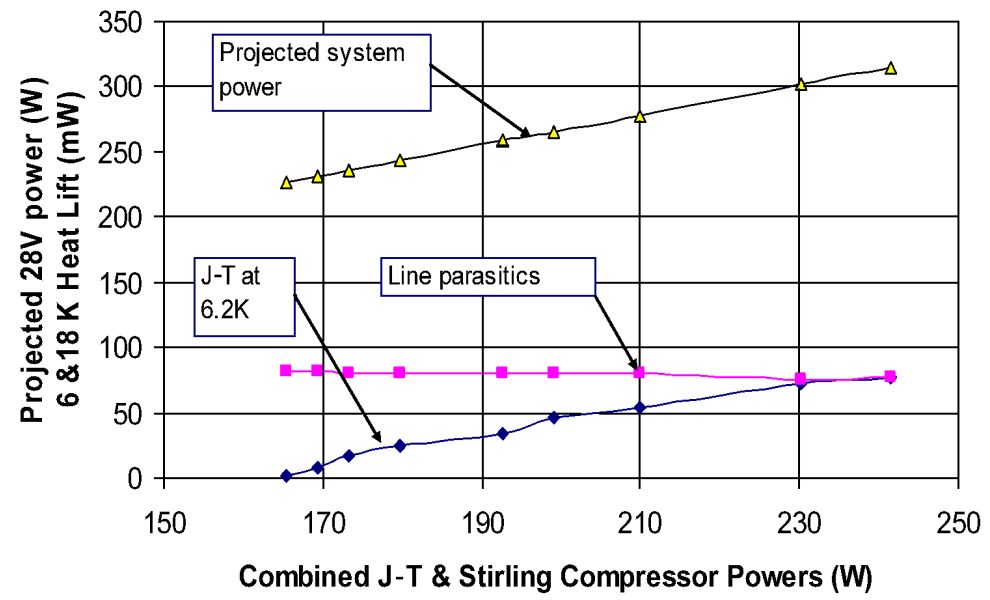

Figure 5. The heat lift at two interfaces: $18 \mathrm{~K}$ representing line parasitics and $6.2 \mathrm{~K}$ representing the refrigeration required by the MIRI instrument for a $285 \mathrm{~K}$ environment. 


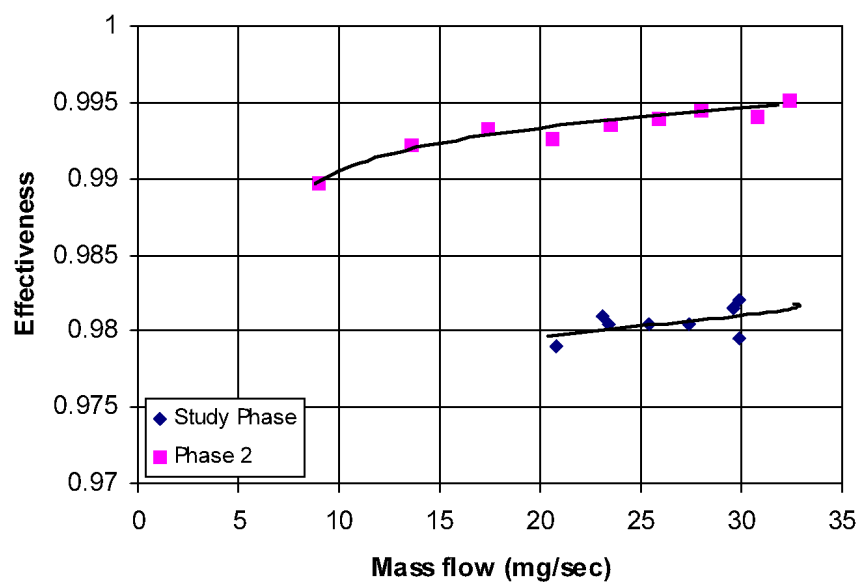

Figure 6. The final version of the ACTDP heat exchanger was well over 99\% effective and its performance contributed to the overall performance of the hybrid cooler.

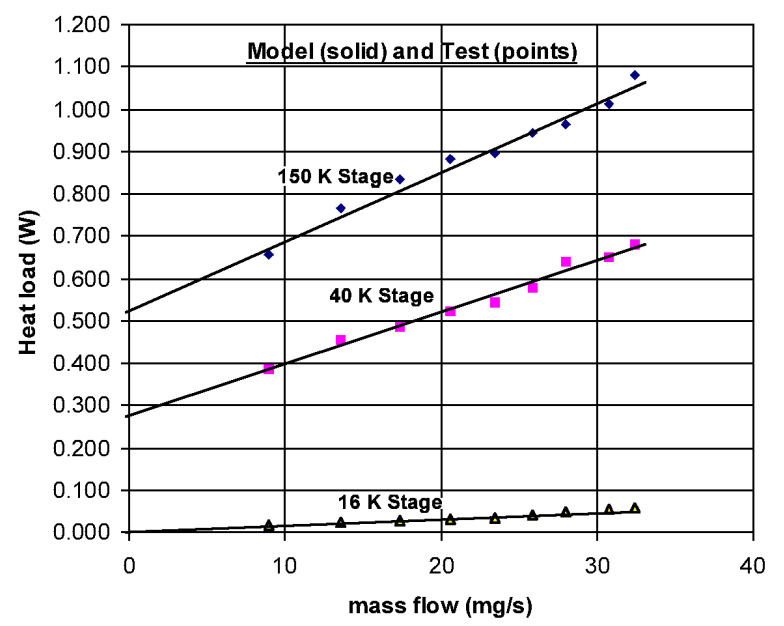

Figure 7. Parasitic loads on the individual stages of our precooler.

poor. To get around this problem the expansion valve can be bypassed by a shunt. With the pressure drop gone, the J-T cooling is replaced by circulating the load back to the precooler. Our initial results in this configuration are shown in Figure 8. Our cooler meets the requirements at high and low temperatures but falls short around $60 \mathrm{~K}$. The deficit amounts to a day's extension of our calculated 2-week cooldown shown in Figure 9. Powers are generally lower in bypass mode since the Stirling is warm and the J-T drives no pressure head.

The J-T cooler's refrigeration holds up pretty well below $6.2 \mathrm{~K}$ as shown in Figure 10. In this test the temperature was allowed to drop at fixed gas charge. Both cooling and input power go down because of the dropping system pressure. The parasitic load carried by the precooler was unchanged. Substituting $3 \mathrm{He}$ has extended the performance to $3.5 \mathrm{~K} .^{2}$

In a final test the cooler was reconfigured to operate at $10 \mathrm{~K}$. For this test the J-T valve impedance was reduced to accommodate the higher temperature, and the system charge pressure was increased by $50 \%$ to better balance the exchanger. As a result of these changes, the existing hardware cooler was able to deliver $135 \mathrm{~mW}$ at $10 \mathrm{~K}$. 


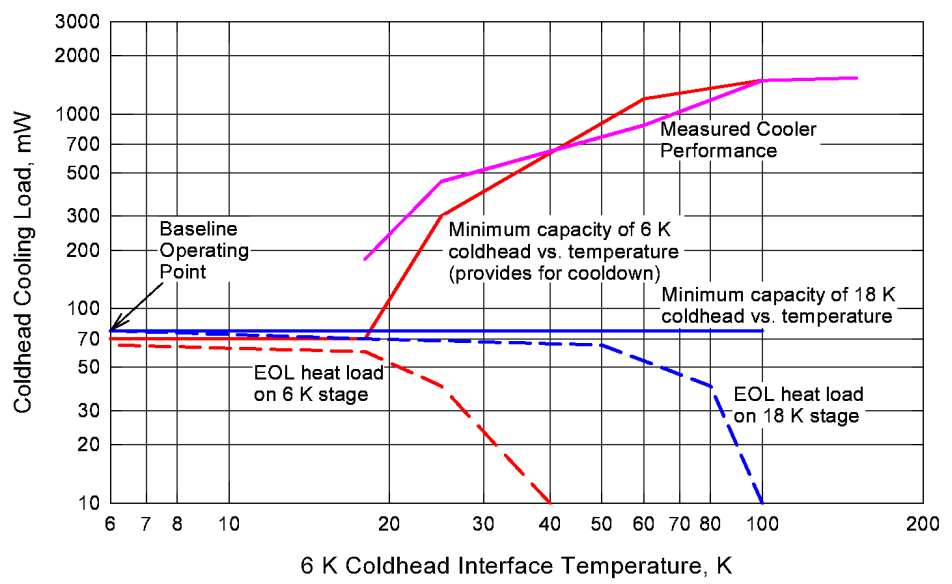

Figure 8. The measured refrigeration at the cold tip at higher temperatures.

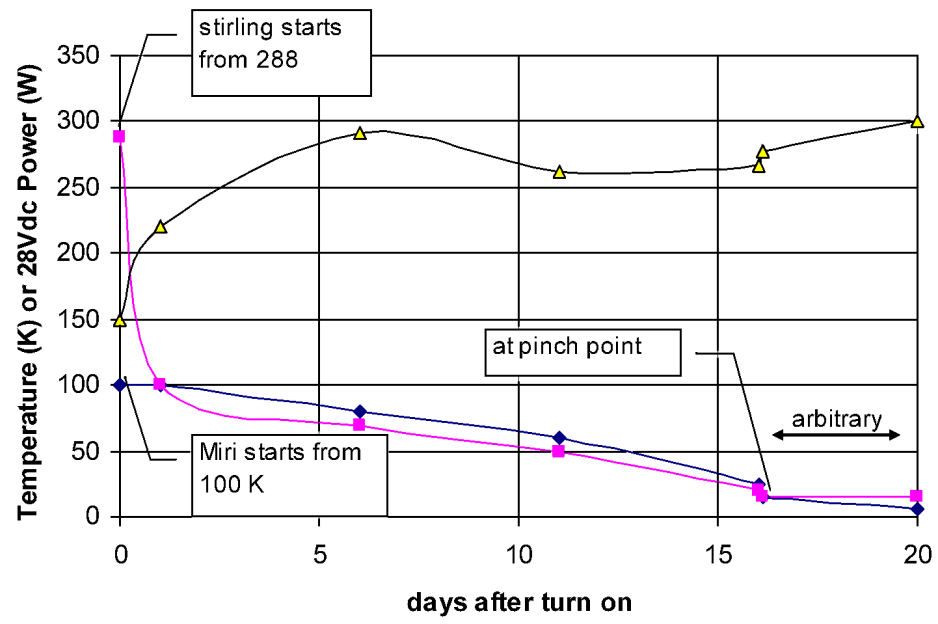

Figure 9. The projected power profile during cool down interpolated from our data.

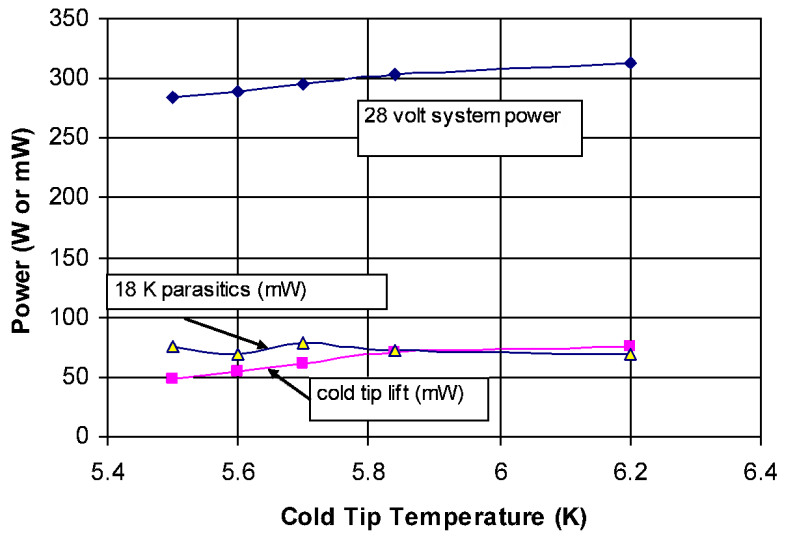

Figure 10. Performance below $6.2 \mathrm{~K}$ at fixed gas charge. 


\section{SUMMARY}

The Ball 4-6 K Hybrid cryocooler has been successfully tested against the proposed MIRI program requirements. The cooler carried the stated heat loads for well under the allocated input power. Detailed characterizations of the individual components were performed. Extensions show the applicability of the system to lower and higher temperatures.

\section{ACKNOWLEDGMENTS}

As always, special thanks goes to Dave Taylor for his expertise in building and assembling Ball cryocoolers. The skill brought to the team by Glen McIntosh of CTS and Bob Levenduski of Redstone Engineering was very welcome and appreciated in this effort. Finally, special thanks to the entire team for their many long hours completing the system test, especially Eric Marquardt.

\section{REFERENCES}

1. Ross, R.G., Jr., "A Study of the Use of 6K ACTDP Cryocoolers for the MIRI Instrument on JWST," Cryocoolers 13, Kluwer Academic/Plenum Publishers, New York (2005), pp.15-30.

2. Glaister, D.S., Gully, W., Ross, Jr., R.G., Stack, R. and Marquardt, E., "Ball Aerospace 4-10 K Space Coolers," Cryocoolers 13, Kluwer Academic/Plenum Publishers, New York (2005), pp. 1-8.

3. Cryogenic Technical Services, 164 Primrose Ct., Longmont, CO 80501.

4. Gully, W., Glaister, D.S., Hendershott, P., Kotsubo, V., Lock, J., and Marquardt, E., "Ball Aerospace Next Generation Two-Stage 35 K Coolers: the SB235 and SB235E," Cryocoolers 14, ICC Press, Boulder, CO, 2007, this proceedings.

5. Gully, W.J., and Glaister, D., “The Ball 12 K Stirling Cryocooler,” Adv. in Cryogenic Engineering, Vol. 47B, Amer. Institute of Physics, Melville, NY (2002), pp. 1045-1052. 